\title{
Visual reaction effects induced and stimulated by different lights on phototactic bio-behaviors in Locusta migratoria manilensis
}

\author{
Liu Qihang ${ }^{1}$, Xin Zhe ${ }^{2}$, Zhou Qiang ${ }^{2 *}$ \\ (1. School of Mechanical and Electrical Engineering, Henan Institute of Science and Technology, Xinxiang 453003, China; \\ 2. College of Engineering, China Agricultural University, Beijing 100083, China)
}

\begin{abstract}
Control of locusts traditionally relies on chemical insecticides. However, chemical control had led to serious environment problems, while light trap techniques based on manipulating insect phototactic responses, are proposed to use for plague locusts control. The key to high efficiency of the trap is to decide the photoelectric reaction intensities on the positive phototaxis of insects. The study compared the visual reaction effects of locusts with their phototactic photosensitivity through the use of spectrometry and behavior investigations, to obtain the influences of the visual spectrum characteristics on locusts positive phototaxis. The results showed that the visual absorption of photons of mixed spectral light and the visual glimmer reaction of the locusts with migratory spectrum characteristics of the peak wavelength of LED lights, reflected the degree of the visual photosensitivity to light photons absorbed by the eye. The visual photosensitive response intensity and the phototactic aggregation effect stimulated by violet light were the most effective, showing that violet light of locusts visual trending sensitivity was consistent with the short sensitive spectrum light of insects visual electrophysiology, whereas the timeliness of the visual photosensitivity reaction and the induction effect was superior under orange light, showing that the biological behavior of locusts visual physiology reaction caused by orange light regulating locusts light rhythm was complicated. Moreover, weak light resulted in stronger visuospatial orientation in terms of the phototaxis of locusts and stronger light resulted in the better visual orientation towards the light source. The results indicated that optical wave properties could affect the visual absorption of spectral light photons, which resulted in different visual responses to different optical frequency energies and restricted the visual bio-photosensitive induction activity of locusts. The study provides new understanding of positive and negative phototaxis of locusts and useful data for the further development of photoelectric induction techniques for the control of locusts.
\end{abstract}

Keywords: locusta migratoria, visual reaction effect, phototactic bio-behavior, light property, bio-control

DOI: $10.25165 /$ j.ijabe.20171004.2357

Citation: Liu Q H, Xin Z, Zhou Q. Visual reaction effects induced and stimulated by different lights on phototactic bio-behaviors in Locusta migratoria manilensis. Int J Agric \& Biol Eng, 2017; 10(4): 173-181.

\section{Introduction}

In nature, many Lepidoptera and Coleoptera show the phototactic behaviors ${ }^{[1,2]}$. Orthoptera, including locusts,

Received date: 2016-01-29 Accepted date: 2017-03-19

Biography: Liu Qihang, PhD, Lecturer, research interests: locust capturing technology of optical-mechanical-electrical integration, Email: bjliuqihang@163.com; Xin Zhe, PhD, Professor, research interests: agricultural machinery and power engineering, CFD. Email: xinzhe@cau.edu.cn.

*Corresponding authors: Zhou Qiang, Professor, research interests: phototaxis trapping control of agriculture pests. Department of Mechanical and Electrical Engineering, College of Engineering, China Agricultural University. Tel: +86-10-62736512, Email: zq@cau.edu.cn. also show certain phototactic behaviors, a conclusion based on the phenomenon of the migration of locusts to urban areas during the night and behavioral determination analyses of the spectral sensitivity of locusts. The visual photoelectric induction effect of locusts has been revealed by analyzing the function of a variety of visual stimuli on the visual nerve system of these insects ${ }^{[3]}$. These results have led to the use of photoelectric induction technologies in the control of these insects ${ }^{[4,5]}$. Such technologies have been used to determine the visual induction triggers of the positive phototaxis behavior of locusts; such information is key to understanding the phototactic aggregation of these insects. In addition, this information has been used to investigate the 
photosensitivity and visual reactions of locusts to spectral light, and to explore the effects of stimulation of the biophotoelectric spectrum of these insects; such studies have the application relevance in that their results can be used to induce the phototactic behavior of these, and other insects.

The spectral sensitivity of locusts has received much attention. The existence of a mix of pigments with $\lambda_{\max }$ of $430 \mathrm{~nm}$ and $515 \mathrm{~nm}$ was firstly determined by intracellular recording of single photoreceptor cells in the compound eye of the locust. Three types of receptor with $\lambda_{\max }$ of $360 \mathrm{~nm}, 430 \mathrm{~nm}$ and $515 \mathrm{~nm}$ were also subsequently identified in the retina of locusts by extracellular recording, and it was assumed that each receptor contained only one light-sensitive pigment; such receptor coupling was thought to have functional advantages in enhancing ability of the insect to detect signals ${ }^{[6,7]}$. Similarly, using intracellular recording, Osorio $^{[8]}$ investigated the influence of ultraviolet (UV) and violet light on the phototactic behavior of locusts, and Farrow $^{[9]}$ showed that violet light can be used to attract locusts using light trap systems. Microspectrophotometry has been used to investigate the mechanics behind the phototactic vision of locusts, such as their spectral sensitivity, light adaptation, visual acuity, and the roles of different visual pigments, such as metarhodopsin. Electroretinogram recordings revealed regular changes in the angular sensitivity of retinula cells in, as well as changes in the physiological structure of, the compound eye of locusts. These findings suggest that the regulation and synergetic action of visual and screening pigments adjusted by the optical field induce the spectral sensitivity of locusts ${ }^{[10,11]}$. The study of the phototactic behaviors of locusts showed that, in response to different light intensities, the dorsal ocelli regulate the speed of movement and the threshold for the initiation of certain activities, and, coupled with the compound eye, are involved in angular orientation, Jander and Barry ${ }^{[12]}$ and Motohiro and Yukiko ${ }^{[13]}$ showed that the dorsal ocelli of locusts controls the response of the compound eye during phototaxis based on the principle of phototactic push-pull coupling.

Despite much research on the visual biophotoelectricity effects and visual reaction of locusts, there has been less focus on the visual response of locusts to different spectral properties of light. Until recently, there had also been little experimental work on the effects of photosensitive excitation on the visual sensitivity behavior of locusts. Therefore, in this current study, we used spectrophotometry to investigate the relation between the visual spectrum performance and the visual reaction of locusts. We also measured the optical behavior characteristics of the phototactic response. The results of our study provide a theoretical basis for explaining the phototactic visual nature of locusts and the application of phototactic-inducing technologies for use in the control of these insects.

\section{Materials and methods}

\subsection{Experimental samples}

Locusts (Locusta migratoria manilensis) were obtained from an artificial breeding base at Cangzhou, Hebei, China, and were maintained in a laboratory colony under photoperiod of L12:D12. The locusts were fed with grass plants from campus. Owing to the better biological activity, locust adults were tested after emerging one week, between 20:00-24:00 at room temperature $\left(27^{\circ} \mathrm{C}-30^{\circ} \mathrm{C}\right)$.

\subsection{Visual responses of locusts to different properties of light sources}

This experiment was carried out with the AvaSpec spectrometer system using mixed spectrum light stimuli provided by AvaLight-DH-S ${ }^{[14]}$ (spectral band range, 215-800 nm; peak wavelength, $400 \mathrm{~nm}$; main spectral wave band, 215-430 nm) and LED light stimuli provided by a $3 \mathrm{~W}$ LED light source, peaking at $400 \mathrm{~nm}, 450 \mathrm{~nm}$, $520 \mathrm{~nm}$ and $610 \mathrm{~nm}$ (Figure 1).

The light beam of the AvaLight-DH-S was focused on the tip of a fiber probe fixed on a stand and was placed close to the compound eye of the insect (Figure 1a); the illumination, stimulating the visual system of the test locust was $100 \mathrm{~lx}$, coming from the natural niche of locust. The LED light source was controlled by a PWM modulation system and driven by a constant $12 \mathrm{~V}$ power supply; it was placed at a set distance of $30 \mathrm{~mm}$ from the visual system of the locust. The illumination from the 
LED light source was measured using an illuminance meter (model-ST-80C) and the PWM modulation system was adjusted to ensure that $100 \mathrm{~lx}$ light stimulated the visual system of the test locust (Figure 1b).

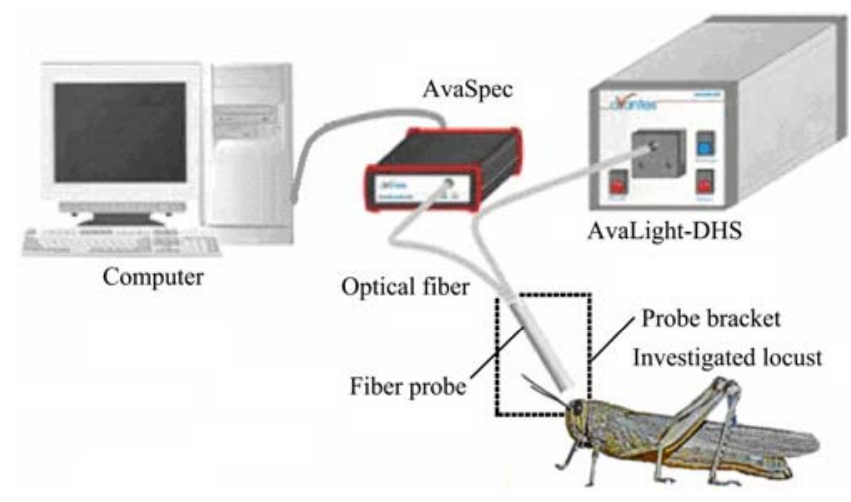

a. Compound eye of the test locust was stimulated by mixed spectrum light provided by AvaLight-DH-S; Fiber probe was a combination of a spectral probe and light fiber probe

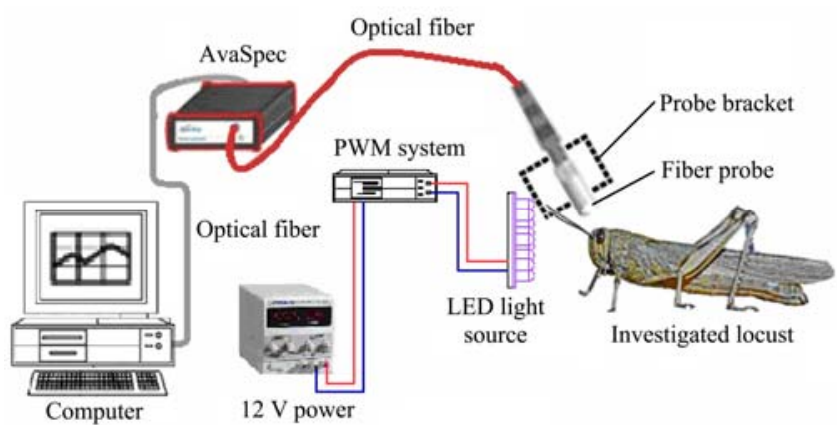

b. Visual system of the locust was stimulated by monochromatic LED light peaking at $400 \mathrm{~nm}, 450 \mathrm{~nm}, 520 \mathrm{~nm}$ and $610 \mathrm{~nm}$; LED light source comprised a

$3 \mathrm{~W}$ LED light source welded in an aluminum substrate and with a $30^{\circ}$ convergent lens

Figure 1 Experimental setup for testing the visual spectrum effect of the phototactic visual response of a locust

For experiments using different light stimulus properties, a locust was fixed with beeswax onto a plastic stage and stimulated with $100 \mathrm{~lx}$ light, as stimulating its visual system. An optical fiber probe connected to the AvaSpec fiberoptic spectrometer system with a probe bracket was placed at a set angle of inclination relative to the horizontal plane to test the response of the compound eye of the locust (Figure 1). The visual response effect stimulated by different (or no) light was recorded using a spectrometer (Model-AvaSpec-ULS2048×64, Spectral testing range: 200-1100 nm) connected to a computer (Figure 1). The recording time of the AvaSpec spectrometer was set to $200 \mathrm{~ms}$.

After leaving the locust for $30 \mathrm{~min}$ without light stimulation, the AvaSpec fiberoptic spectrometer was corrected on the basis of the visual state of the locust, to avoid any effects of natural light on the test results; the result of testing the visual response effect with no light stimulation was recorded. To test for visual responses to different spectral effects, the eye of the locust (Figure 1a) was stimulated with $100 \mathrm{~lx}$ mixed spectrum light provided by the AvaLight-DH-S, for 5 min, $10 \mathrm{~min}$, $20 \mathrm{~min}, 30 \mathrm{~min}, 40 \mathrm{~min}, 50 \mathrm{~min}$ and $60 \mathrm{~min}$, with $30 \mathrm{~min}$ interval between each exposure time. The visual system of the locust (as shown in Figure 1b) was then stimulated with $100 \mathrm{~lx}$ violet, orange, green, or blue light provided by a $3 \mathrm{~W}$ LED light source, with 30 min interval between each exposure. The length of exposure time was based on the results of the visual spectrum performance induced by mixed light. The AvaSpec fiberoptic spectrometer was used to determine the spectral response effect in real time by turning off the light source after the illumination time had ended. The difference between the visual response characteristics with and without light was then determined.

\subsection{Behavior responses of locusts to light intensity and spectrum}

Behavioral verification experiments of the response of the locust visual system to violet, orange, green, and blue light were carried out in a dark room at $27^{\circ} \mathrm{C}-30^{\circ} \mathrm{C}$ using a 3.5 m-long straight channel comprised of opaque acrylic plates (Figure 2).

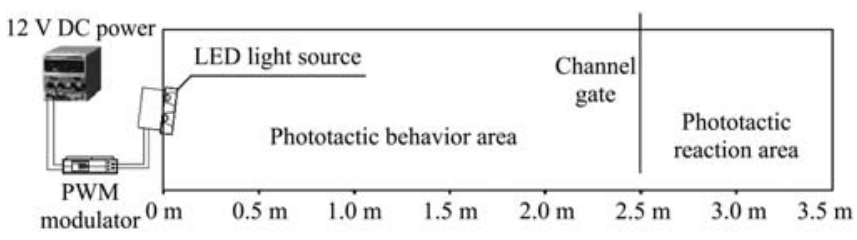

Figure 2 Experimental setup for verifying the degree of visual response of the locust to an LED spectral light gradient

The channel was divided into two areas at $2.5 \mathrm{~m}$ (Figure 2): (i) the phototactic behavior area relating to the visual response of the locust to light stimuli, and (ii) the phototactic reaction area. Access between the two areas was provided by a black gate. The end of the phototactic reaction area was sealed with a black plate, whereas the phototactic behavior area was sealed with a black plate containing a $3 \mathrm{~W}$ LED light source in its center at a $10^{\circ}$ angle, with a $12 \mathrm{~V}$ DC power supply and controlled by a PWM modulator to maintain constant light illumination; this was to avoid any nonlinear relations between the 
LED current and light intensity affecting our results.

LED spectral stimuli were provided with peak wavelengths at $400 \mathrm{~nm}, 450 \mathrm{~nm}, 520 \mathrm{~nm}$ and $610 \mathrm{~nm}$. The stimuli were calibrated by an illuminance meter (model-ST-80C) and controlled by a PWM modulator to a set $100 \mathrm{~lx}$ (Table 1). Seven sections were marked along the length of the channel from left to right, spaced $0.5 \mathrm{~m}$ apart, and were used to observe and distinguish the visual response of the locust to the LED light gradient from the top of the open channel.

Table 1 Visual photosensitivity response distribution of locusts to different spectral light levels

\begin{tabular}{|c|c|c|c|c|c|c|c|c|}
\hline \multirow{2}{*}{$\begin{array}{c}\text { Channel } \\
\text { section/m }\end{array}$} & \multicolumn{4}{|c|}{ Nominal illumination of LED light source (100 lx) } & \multicolumn{4}{|c|}{ Normal illumination of LED light source } \\
\hline & Violet & Orange & Green & Blue & Violet (2000 lx) & Orange (43 $100 \mathrm{~lx}$ ) & Green (64 600 lx) & Blue (10 $000 \mathrm{~lx})$ \\
\hline $0.0-0.5$ & $38.75 \pm 2.5$ & $25.00 \pm 2.5$ & $27.50 \pm 1.25$ & $25.00 \pm 2.5$ & $72.50 \pm 1.25$ & $61.25 \pm 2.5$ & $60.00 \pm 1.25$ & $43.75 \pm 2.5$ \\
\hline $0.5-1.0$ & $7.50 \pm 3.75$ & $13.75 \pm 1.25$ & $15.00 \pm 2.5$ & $12.50 \pm 2.5$ & $3.75 \pm 3.75$ & $20.00 \pm 3.75$ & $7.50 \pm 2.5$ & $12.50 \pm 3.75$ \\
\hline $1.0-1.5$ & $7.50 \pm 2.5$ & $17.50 \pm 2.5$ & $18.75 \pm 1.25$ & $16.25 \pm 3.75$ & $8.75 \pm 2.5$ & $16.25 \pm 2.5$ & $8.75 \pm 2.5$ & $8.75 \pm 2.5$ \\
\hline $1.5-2.0$ & $15.00 \pm 1.75$ & $20.00 \pm 1.25$ & $20.00 \pm 2.5$ & $18.75 \pm 2.5$ & $8.75 \pm 3.75$ & $0.00 \pm 2.5$ & $11.25 \pm 2.5$ & $7.50 \pm 2.5$ \\
\hline $2.0-2.5$ & $15.00 \pm 2.5$ & $16.25 \pm 2.5$ & $10.00 \pm 2.5$ & $18.75 \pm 3.75$ & $2.50 \pm 2.5$ & $0.00 \pm 2.5$ & $3.75 \pm 2.5$ & $6.25 \pm 1.25$ \\
\hline
\end{tabular}

Note: Data are mean \pm SE of three trials.

The phototactic device comprised a straight channel divided by a gate into a $2.5 \mathrm{~m}$ long phototactic behavior area and a $1 \mathrm{~m}$ long phototactic reaction area. The length, width and depth of the channel were $3.5 \mathrm{~m}, 0.5 \mathrm{~m}$, and $0.5 \mathrm{~m}$, respectively. Two black plates sealed each end of the channel. LED light source was positioned in the center of the left plate to provide light stimulation.

To test the visual response of locusts to monochromatic light with a set illumination, 640 individual locusts were divided into eight groups (80 in each), and each test combination of light wavelength and spectral illumination used one group. Before starting each test, the phototactic behavior area and the phototactic reaction area were separated by the channel gate. The LED light source, at a set $100 \mathrm{~lx}$ or nominal illumination, as determined by the PWM modulator, was turned on. One group of 80 locusts was released into the phototactic reaction area and kept for 30 min in the dark (i.e., without any LED spectral light stimulus). The channel gate was then opened for $10 \mathrm{~min}$. Each group was tested three times in a row; each wavelength (violet, orange, green or blue) at $100 \mathrm{~lx}$ was tested with each of the eight groups three times, before moving on to the next wavelength. In each experiment, the stagnant response times of the locusts in the $0.5-1.0 \mathrm{~m}$ and $2.0-2.5 \mathrm{~m}$ sections of the channel were recorded during the $10 \mathrm{~min}$ stimulation time. To avoid the error caused by random action of locust, after each experiment, the gate was opened to keep the channel with 10 min dark, then the gate was closed to take a record of locust numbers in different section and the mean number of locusts in each test in each section was calculated.

The mean percentage of locusts in each section was used to reflect the photosensitive response degree of the insects to different spectral light illuminations; the mean stagnant response times of the locusts in the $0.5-1.0 \mathrm{~m}$, 2.0-2.5 $\mathrm{m}$ sections were used for the degree of photosensitive movement regulated by light and the visual orientation response of the locusts induced by the timing of light stimulation, respectively.

\section{Results and discussion}

\subsection{Visual response of locusts to mixed spectral light and LED spectral light}

After the compound eye was exposed for different lengths of time to $100 \mathrm{~lx}$ mixed spectral light stimulation (or no light stimulation), we immediately measured the visual response spectrum. The superposed results of visual spectrum effect, presented in Figure 3 was the spectral effect of the visual response of locust with light stimulation minus that with no light, with a secondary concave peak at $545 \mathrm{~nm}$ and a tertiary concave peak at $435 \mathrm{~nm}$, could be fitted by the sum of the absorption spectra of the three putative visual pigments recorded in locusts $^{[15]}$ with absorption maxima at $360 \mathrm{~nm}, 430 \mathrm{~nm}$ and $530 \mathrm{~nm}$, respectively. However, the primary absorption peak at $610 \mathrm{~nm}$ did not correspond to the spectral sensitivity of UV, B, and G receptors in 
compound eye of the locust. The absorption by the compound eye of spectral photons at $435 \mathrm{~nm}, 540 \mathrm{~nm}$ and $610 \mathrm{~nm}$ showed an incremental trend with increasing stimulation time, and the absorption degree was the strongest at $50 \mathrm{~min}$, whereas the visual absorption response at $60 \mathrm{~min}$ tended to be the steady-state absorption rate. These results showed that visual system of the locust has sensitive and absorptive selectivity in response to different spectral light stimuli.

Based on an analysis of the results shown in Figure 3, we investigated the visual response spectrum of the visual system of the locust induced by different LED spectral intensities with a stimulation time of 50 min through minus that with no light stimulation (Figure 4).

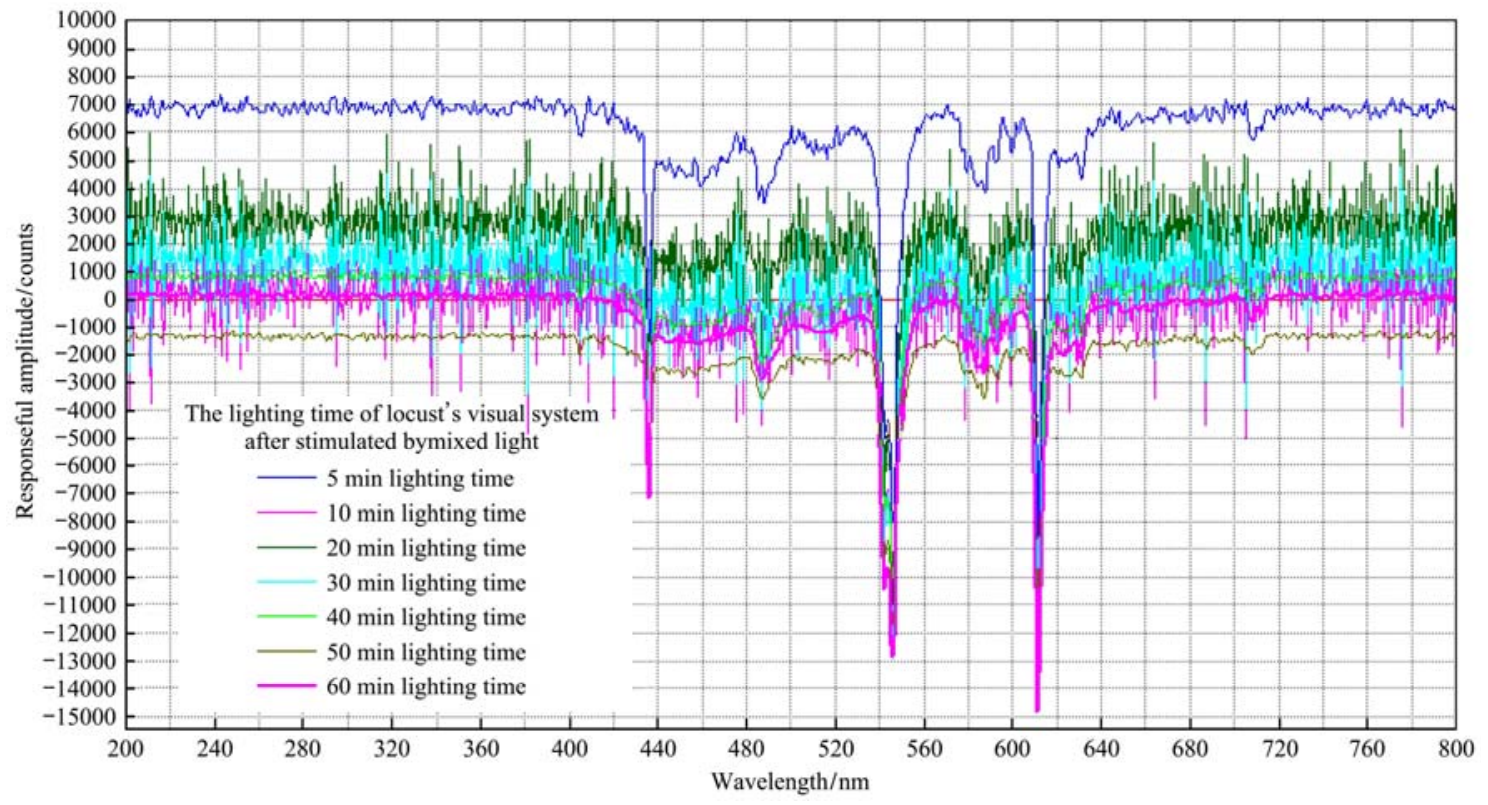

Figure 3 Spectral effects of the visual response of locust with light stimulation minus that with no light (Different colored lines correspond to different stimulation times)

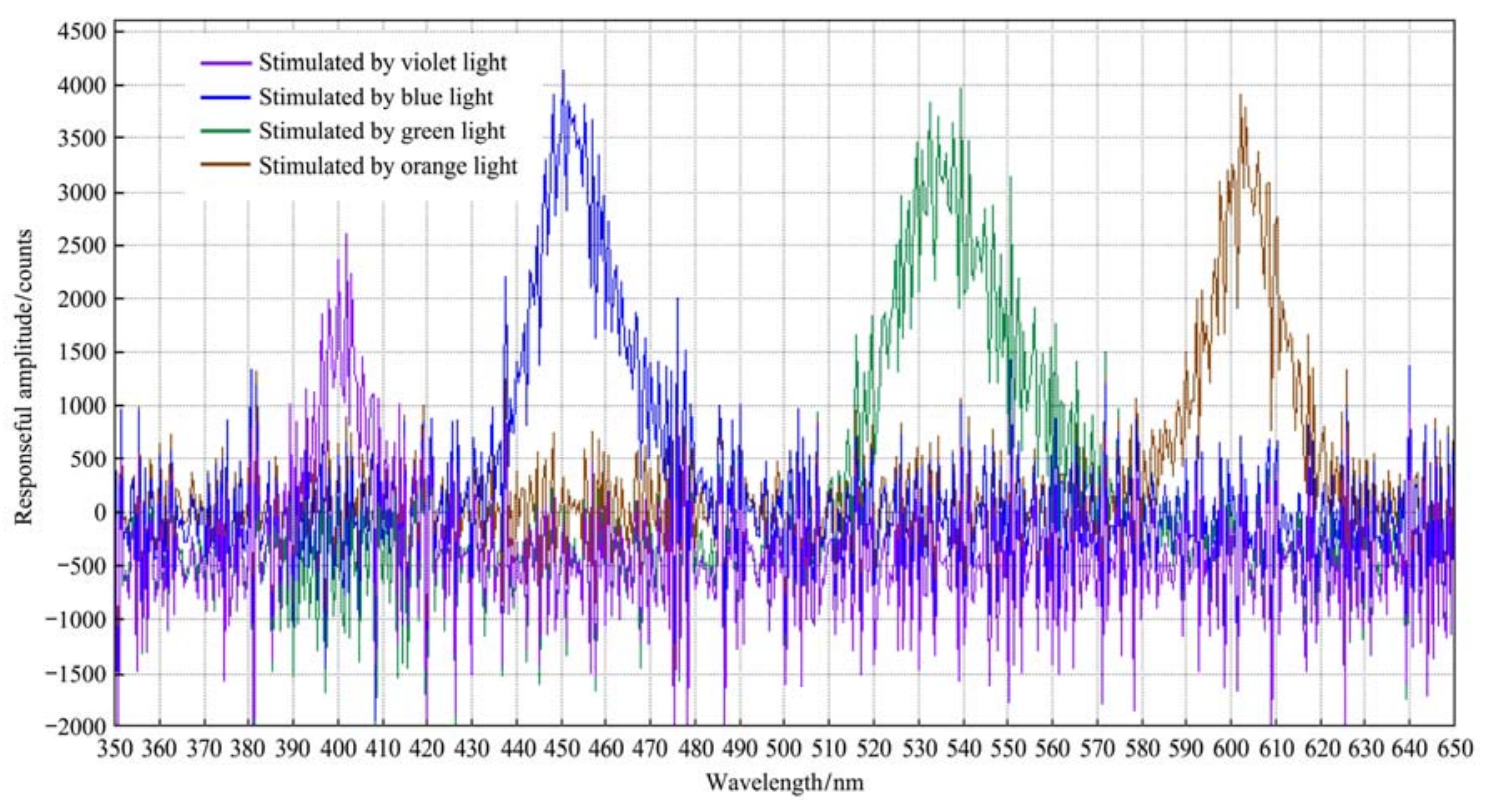

Figure 4 Visual responses of the visual system of the locusts to 50 min stimulation at different LED light wavelengths through minus that with no light stimulation (Different colored lines correspond to the different spectral light stimulations)

Figure 4 shows that stimulation by monochromatic LED light evoked the visual spectrum effect of the response of the locusts to violet, blue, green and orange light, with wavelength peaks at $402 \mathrm{~nm}, 450 \mathrm{~nm}, 540 \mathrm{~nm}$, and $602 \mathrm{~nm}$ respectively, showing that there was a shift in the visual response peak wavelengths relative to the main wavelengths (400 nm, $450 \mathrm{~nm}, 520 \mathrm{~nm}$, and $610 \mathrm{~nm}$ ) of LED light stimulation with the same stimulating time. 
The highest wavelength peak in the visual response and the lowest response were induced by blue and violet light, respectively. These results showed that LED lights could be used to induce biophotoelectric effects in locusts visual system.

When a more detailed analysis was performed, the visual system of the locusts, after stimulation with mixed spectral light, strongly absorbed green and orange photons (Figure 4); after stimulation with green or orange LED light, the peak wavelength of the visual glimmer response, corresponding to the main wavelengths of green or orange light (520 nm or $610 \mathrm{~nm}$ ), increased by $20 \mathrm{~nm}$ and decreased by $8 \mathrm{~nm}$, respectively. The peak wavelength of the visual glimmer response to the main wavelength $(450 \mathrm{~nm})$ of blue light did not shift, whereas the response to the main wavelength $(400 \mathrm{~nm})$ of violet light shifted by $2 \mathrm{~nm}$. Thus, it seems that the difference in visual photoelectric effect between the absorption reaction and the glimmer response must be due to the different light properties of mixed spectral light and monochromatic spectral lights. By contrast, the difference in peak wavelength offset and the glimmer response intensity of the visual response of the locusts to different monochromatic spectral lights must be due to the difference in visual photosensitive reaction at different spectral light stimulations, thus affecting the degree of biological activity of the visual response of the insect.

\subsection{Phototactic photosensitivity of locusts in response to LED spectral light}

We investigated the visual photosensitivity response distribution of locusts to monochromatic violet, orange, green and blue light under two illuminations (100 lx versus nominal illumination) and the results are shown in Table 1. For example, using an LED violet light source at $100 \mathrm{~lx}$ versus $2000 \mathrm{~lx}$ (nominal illumination), the percentage mean number of locusts in the 0.0-0.5 m section of the channel was $38.75 \%$ and $72.50 \%$, respectively. In addition, we recorded the length of inactivity of the visual response of the locust to the light source in the different sections of the experimental channel (0.5-1.0 $\mathrm{m}$ and 2.0-2.5 $\mathrm{m})$, and the results are shown in Figure 5.

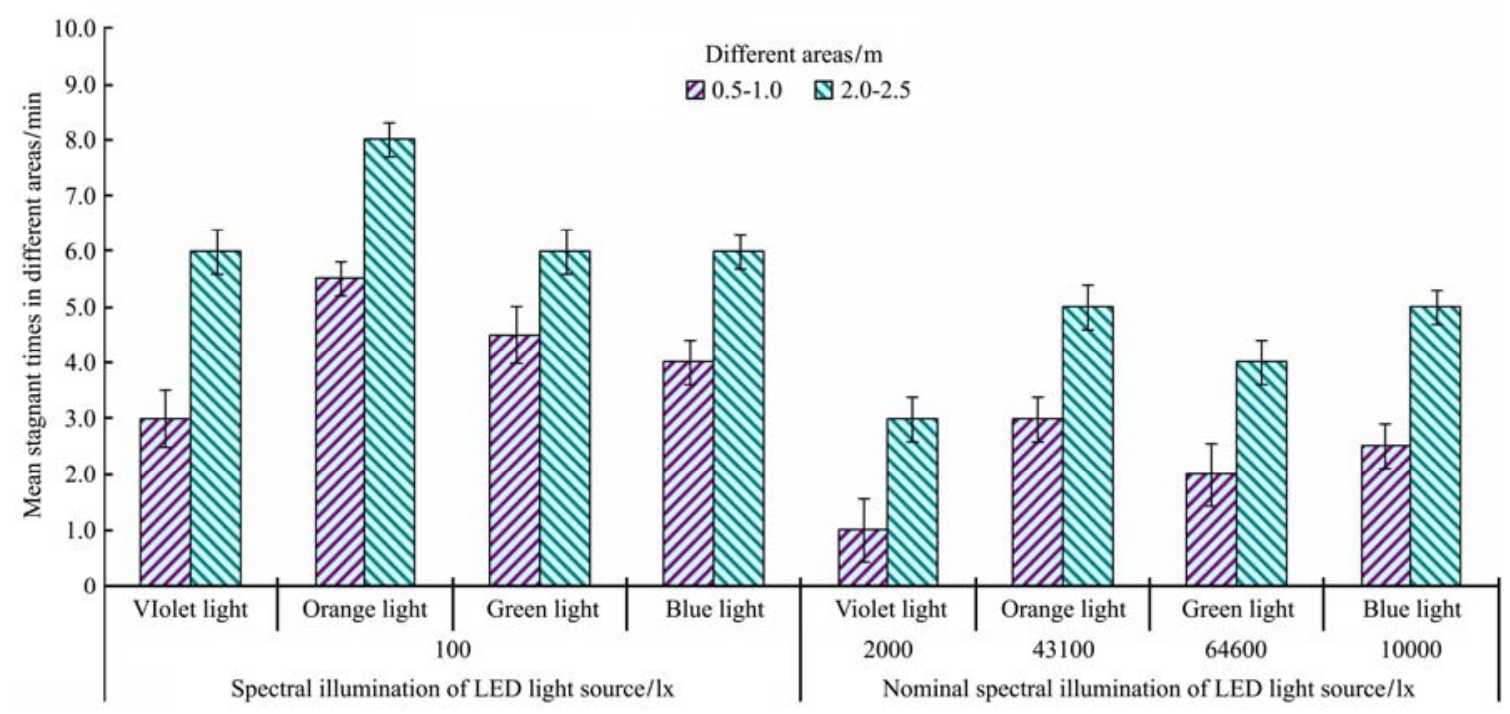

Figure 5 Mean stagnant times of locusts visual photosensitivity response to different spectral illuminations of LED light sources (Bars with right sloping lines and bars with left sloping lines represent the mean stagnant response time of locusts for different spectral illumination in the channel of 0.5-1.0 $\mathrm{m}$ area and 2.0-2.5 $\mathrm{m}$ area, respectively. Data are mean $\pm \mathrm{SE}$ of three trials)

Different LED spectral light intensities induced the visual photosensitivity of the locusts to generate phototactic activity, but the degree of induction differed with the light intensity, resulting in a significant difference $(F, p<0.025)$ in the number of locusts in different sections of the channel (Table 1).

When the illumination of LED light source was dim
(100 lx), the locust always responded the least to blue light, regardless of where they were in the channel. At 0.0-0.5 $\mathrm{m}$ and $0.0-1.0 \mathrm{~m}$ areas, locusts were the most abundant under violet light, followed by the green and then orange lights, and the difference between violet light and green light was very obvious $(F, p>0.05)$, while the difference between green light and blue light was no 
obvious $(F, p<0.01)$. By contrast, at $0.0-2.5 \mathrm{~m}$, locusts were in the most activity under orange light, followed by green and then violet, and the difference between them was very obvious $(F, p>0.05)$. Locusts that responded to violet light in either the $0.5-1.0 \mathrm{~m}$ or $2.0-2.5 \mathrm{~m}$ sections were the first to stop their phototactic activities, followed by those responding to green, blue and then orange light, and the difference between them is significant $(F, p>0.05)$ (Figure 5).

When the LED light source was increased to rated illumination, the locusts again responded the least to blue light. At 0.0-0.5 m, they responded the most to violet light, followed by orange and green light, and the difference between violet light and orange light was very obvious ( $F, p>0.05$ ), while the difference between orange light and green light was obvious $(F, p=0.01)$. By contrast, at $0.0-1.0 \mathrm{~m}, 1.0-1.5 \mathrm{~m}$ and $0.0-2.5 \mathrm{~m}$, locusts responded most to orange light, followed by the violet and then green light, at $0.0-1.0 \mathrm{~m}$ and at $1.0-1.5 \mathrm{~m}$, the difference between them was very obvious $(F, p>0.05)$, while at 0.0-2.5 m, the difference between orange light and violet light was obvious $(F, p=0.025)$ while the difference between green light and violet light was no obvious $(F, p<0.025)$, and the locusts responded in an obvious manner to the light source. Compared with their responses to illumination at $100 \mathrm{~lx}$, the response time of the locusts was shorter under nominal illumination, being shortest in response to violet light, followed by green, blue and orange light, and the difference among violet light, green light and orange light was very obvious $(F, p>0.05)$, while the difference between orange light and blue light was no obvious $(F$, $p<0.01$ ). These results show that increasing the illumination enhances the bioactivity of the locust and accelerates the acutity of its visual response.

By comparing the visual photosensitivity response distribution with the response time of locusts in the 0.5-1.0 $\mathrm{m}$ and 2.0-2.5 m sections, it can be seen that, under weak illumination at $100 \mathrm{~lx}$, there was no significant difference $(F, p=0.025)$ in the degree of phototactic photosensitivity of the locusts stimulated by orange, green, or blue light; similarly, the response times of locusts induced by violet, green or blue light were the same, showing no significant $(F, p=0.01)$, whereas there were significant differences $(F, p=0.05)$ compared with the response times to orange light. Furthermore, under nominal illumination, the degree of phototactic photosensitivity of locusts stimulated by orange, green and blue light showed obvious differences $(F, p>0.025)$, and the response time of locusts induced by violet, green, or blue light (the response times to blue and orange light were the same) showed significant differences $(F$, $p>0.05$ ).

These results showed that short wave spectrum of violet light could quickly evoke locusts visual response activity to cause the stronger aggregation effect, the long wave spectrum of orange light could induce the visual bio-physiology response to cause the better phototactic effect.

\subsection{Discussion}

The absorption peak wavelength ranges of the visual system of insects are 510-570 nm, 410-490 nm and 340-365 nm; the visual pigment in insects is rhodopsin, which is able to absorb light from the green end of the spectrum through to the UV end, ensuring that insects attain maximum sensitivity to spectral light ${ }^{[16]}$. Thus, locusts and other insects are able to adapt and respond to changes in the intensity of light in their environment ${ }^{[17,18]}$. However, locusts display varying levels of sensitivity to different spectral light levels, which can affect the phototactic behavior of the insect ${ }^{[19]}$.

Our study of the visual spectrum of the locust (Figures 3 and 4) shows the degree of influence of different spectral light on the visual reaction of these insects. Different spectral light properties resulted in different visual responses with corresponding spectral effects; that is, mixed light with a wide wave band caused the eye to absorb sensitive light photons, whereas LED spectral light with a narrow wave band stimulated the visual system to induce the glimmering reaction with characteristics of a mobility spectrum peak value. Thus, optical frequency energy and optical wave properties affect the photosensitive reaction degree of locusts visual activity, and the inspiring effect of LED spectral light on the visual system of locusts was stronger. The difference in the visual absorption response to sensitive 
light energy, and the spectral migratory difference between the peak wavelength of the visual response and the peak wavelength of the stimulating light, is likely to be due to the photosensitive mediation effect of the visual system of insect inhibiting and activating the excitatory activity of the visual response, indiating that the properties of spectral light quality induce different visual physiology reaction effect of insects. This affects the stimulation of biophotoelectric effects and the response intensity of phototactic biophysiological activity.

The results of the phototactic photosensitivity of the response of the locusts to LED spectral light (Table 1 and Figure 6) support the results on the effect of LED spectral light on the visual response of these insects. The results showed that only stimulation of the visual spectrum reaction can result in phototactic response. Under weak light (100 lx), the conversion of the visual reaction to a visual response was longer and the selectivity of the locust to the illumination of the light source was weaker comparing with the stronger light. Moreover, the photosensitization of the visual response stimulated by violet light was the strongest, and the photosensitive response velocity induced by orange light was the slowest, this resulted in locusts aggregating more under violet light, whereas orange light had more of an reaction effect, showing that locusts visual physiology excitability stimulated by violet light is the stronger and the regulation effect of locusts visual physiology rhythm induced by orange light is the better. Under nominal illumination, there was less time between the visual reaction and the visual response and the selectivity of locusts to the light source was stronger, resulting in more prominent phototactic aggregation. Thus, weak photosensitivity responses indicated that, under weak light, locusts rely on visual orientation functions to generate the phototactic identification of their light environment, manifested by slow phototaxis and a poor degree of photosensitivity, whereas strong light disturbs the visual orientation function to generate a bad visual adjustment effect, manifested by fast phototaxis and strong phototactic sensitivity.

Thus, effects on the visual spectrum of a locust induced by LED spectral light reflect the degree of the visual reaction of the insect to the spectral photon absorbed by the eye, embodied by the differences in photosensitive induction of the visual response of the locust to different spectral light energies. This indicates that generation of locusts visual physiology excitability and visual physiology rhythm stimulated and regulated by different spectral light levels affects the biophotoelectric sensitivity and the phototactic selectivity of these insects, manifested by differences in the phototactic response and visual regulation reaction under different spectral light levels.

\section{Conclusions}

Mixed spectral light stimulates the compound eye of locusts to absorb light photons with peak wavelengths of $430 \mathrm{~nm}, 545 \mathrm{~nm}$ and $610 \mathrm{~nm}$. LED monochromatic light with peak wavelengths of $400 \mathrm{~nm}, 610 \mathrm{~nm}$ and $520 \mathrm{~nm}$ stimulates the visual system of locusts to generate a glimmer reaction with peak wavelengths of $402 \mathrm{~nm}$, $602 \mathrm{~nm}$ and $540 \mathrm{~nm}$. By contrast, blue light stimulation did not have any effect on the visual system. In addition, visual spectral emission amplitude stimulated by LED spectral light reflects the degree of the visual photosensitive reaction to light photons absorbed by the eye. This study of the phototactic photosensitivity effect demonstrated that only stimulation of the visual spectrum reaction resulted in a phototactic response and that violet light resulted in stronger photosensitivity, resulting in more prominent phototactic aggregation. Orange light stimulated a slower photosensitive response, which had a better regulation effect and elicited a good induction effect. Moreover, optical wave properties affected the degree of visual absorption of spectral light photons and the generation of different glimmer responses stimulated by different optical frequencies, restricting generation of activity induced by visual photosensitive responses.

\section{Acknowledgment}

We acknowledge that this research was financially supported by 2012 Public Welfare Industry (Agriculture) Special Funds for Scientific Research Projects (Grant No. 201203025), Scientific and Technological Project in Henan Province (Grant No.172102110270), and the Landmark Innovation Project of Science and Technology. 


\section{[References]}

[1] Jiang X, Mai T M, Zhang L. Nocturnal migration of grasshopper (Acrididae: Oedaleus asiaticus). Acta Agrestia Sinice, 2003; 11(3): 75-77.

[2] Wei G S, Zhang Q W, Wu W G. Characteristic response of the compound eyes of Helicoverpa armigera to light. Acta Entomologica Scinica, 2002; 45(3): 323-328.

[3] Barry C K, Jander R. Photoinhibitory function of the dorsal ocelli in the phototactic reaction of the migratory locust. Nature, 1968; 217(5129): 675-677.

[4] Zhou Q, Xu R Q, Cheng X T. Bio-photo-electro effect of insects and its application in pest control. Progress in Modern Biomedicine, 2006; 6(4): 70-72.

[5] Jundi B, Homberg U. Receptive field properties and intensity response functions of polarization-sensitive neurons of the optic tubercle in gregarious and solitarious locusts. J Neurophysiol, 2012; 108: 1695-1710.

[6] Bennett R R, Horridge G A. Spectral sensitivity of single retinula cells of locust. Z. Vergl. Physiology, 1967; 5(12): 195-206.

[7] Osorio B. Ultraviolet sensitivity and spectral opponency in the locust. J. Exp. Biol, 1986; 122: 193-208.

[8] Farrow R. A modified light-trap for obtaining large samples of night-flying locusts and grasshoppers. J. Aust. Ent. Soc, 1974; 13: 357-360.

[9] Jiang J L. Spectral sensitivity of locust compound eyes: A comparative study. Acta Physiologica Sinica, 1983; 35(1): 5-9.

[10] Wu W G, Horridge C A. Regular change of the angular sensitivity of the retinula cells in locust compound eye. Acta Biophysica Sinica, 1987; 3(6): 178-184.

[11] Thomas A M, Rava A S, Sandra S. Approach sensitivity in the retina processed by a multifunctional neural circuit. Nature, 2009; 10(12): 201-211.

[12] Jander R, Barry C K. The phototactic push-pull-coupling between dorsal ocelli and compound eyes in the phototropotaxis of locusts and crickets. Zeitschrift für Vergleichende Physiologie, 1968; 57(4): 432-458.

[13] Motohiro W S, Yukiko M. Physiological basis of phototaxis to near-infrared light in Nephotettix cincticeps. J Comp Physiol A, 2014; 200(13): 527-536.

[14] Liu Q H, Zhou Q. Physiological response of locusts to eye stimulation by spectral illumination for phototactic pest control. Int J Agric \& Biol Eng, 2016; 9(2): 186-194.

[15] Vishnevskaya T M, Cherkasov A D. Spectral sensitivity of photoreceptors in the compound eyes of the locust compound eyes. The Journal of Comparative Neurology, 1983; 35(1): 9-15.

[16] Liu Q H, Zhou Q. Comparative investigation of locust's phototactic visual spectrum effect and phototactic response to spectral illumination. Spectroscopy and Spectral Analysis, 2014; 34(7): 1593-1596.

[17] Michiyo K P, Uwe H. Spectral properties of identified polarized-light sensitive interneurons in the brain of the desert locust Schistocerca gregaria. Journal of Experimental Biology, 2007; 210(8): 1350-1361.

[18] Wang L X, Niu H L, Zhou Q. Locust induced trapping experiment based on coupling effect of air disturbance stimulation and spectrum light source. Transactions of the CSAE, 2014; 30(5): 108-115. (in Chinese)

[19] Liu Q H, Zhou Q. Effect of locust's phototactic response to regulatory illuminance of LED light source. Transactions of the CSAM, 2014; 44(9): 243-249. (in Chinese) 